\title{
EL TIEMPO DE DOBLAJE DE PSA COMO NUEVO MÉTODO DIAGNÓSTICO Y PRONÓSTICO DEL CÁNCER DE PRÓSTATA.
}

\author{
Carlos Llorente, José Manuel de la Morena y Manuel Álvarez Ardura.
}

Servicio de Urología. Fundacion Hospital Alcorcón. Profesor Asociado de Cirugía Universidad Rey Juan Carlos. Madrid. España.

\begin{abstract}
Resumen.- OBJETIVO: Evaluar el papel de la cinética del PSA en el diagnóstico y prosnóstico del cáncer de próstata.

MÉTODOS: Revision sistemática de la literatura a través de Medline.

RESULTADOS: Una cifra de PSAV $2 \mathrm{ng} / \mathrm{ml} /$ año se asocia a una mayor mortalidad por cáncer de próstata tras cirugía o radioterapia. Un PSADT $<3$ meses tras el tratamiento confiere un mal pronóstico y un PSADT>10 meses tras fallo bioquímico despues cirugía situa a la recidiva local como causa más probable.
\end{abstract}

Palabras clave: Cáncer de próstata. PSA. Diagnóstico.
Carlos Llorente Servicio de Urología Fundación Hospital Alcorcón Budapest, 1 28922 Alcorcón. Madrid. (España) cllorente@fhalcorcon.es
Summary.- OBJECTIVES: To review the value of PSA kinetics in the diagnosis and prognosis of prostate cancer.

METHODS: Review of the literature through a Medline search.

CONCLUSIONS: A pre-tretament PSAV value >2ng/ $\mathrm{ml} / \mathrm{yr}$ is a risk factor for increased mortality from prostate cancer after surgery or radiation therapy. A PSADT $<3 \mathrm{~ms}$ is indicative of reduced survival after treatment. For patients with PSA recurrence after surgery a PSADT>10 is more likely associated with local recurrence.

Keywords: Prostate cancer. PSA. Diagnosis.

\section{INTRODUCCIÓN}

La determinación del antígeno específico de la próstata (PSA) se viene utilizando desde la década de los ochenta fundamentalmente dirigida hacia el diagnóstico de cáncer de próstata. La disponibilidad clínica del PSA ha producido un importante incremento en la incidencia de esta enfermedad y ha abierto un profundo debate en cuanto a la conveniencia de la utilización del PSA en el cribado de esta enfermedad.

Poco tiempo después de la incorporación de esta proteína a la actividad clínica se estudió su papel en la estadificacion del cáncer de próstata, incorporándose recientemente en nomogramas predictores de las características histopatológicas de la enfermedad o de los resultados del tratamiento quirúrgico o radioterápico. 
El cáncer de próstata, a diferencia de otras neoplasias, es una enfermedad de larga evolución lo que hace que los resultados de los ensayos clínicos cuya variable principal es la mortalidad específica requieran un largo tiempo de seguimiento $y$, por ello, los clínicos interesados han de esperar también durante un tiempo que excede el de la inactividad clínica ante el proceso de toma de decisiones frente a los enfermos. Por esta razón, en la actualidad se busca con interés la disponibilidad de una variable intermedia de la mortalidad específica. Una de estas variables podría ser la cinética del PSA, en especial, el tiempo de duplicidad del PSA (PSADT).

\section{DEFINICIÓN Y MEDIDA DE CINÉTICA DEL PSA}

La cinética del PSA son los variaciones de PSA a lo largo del tiempo. Esta variación se puede expresar de diferentes maneras como es el tiempo de duplicación del PSA (PSADT) la velocidad del PSA (PSAV) y el log PSA.

EI PSADT indica el tiempo que transcurre para que el PSA doble su valor. Su medición es objeto de controversia en cuanto al método a utilizar. Habitualmente, se determina mediante una ecuación resultante de dividir el logaritmo de $2(0,693)$ por la pendiente de la regresión lineal del logaritmo de PSA en el tiempo.

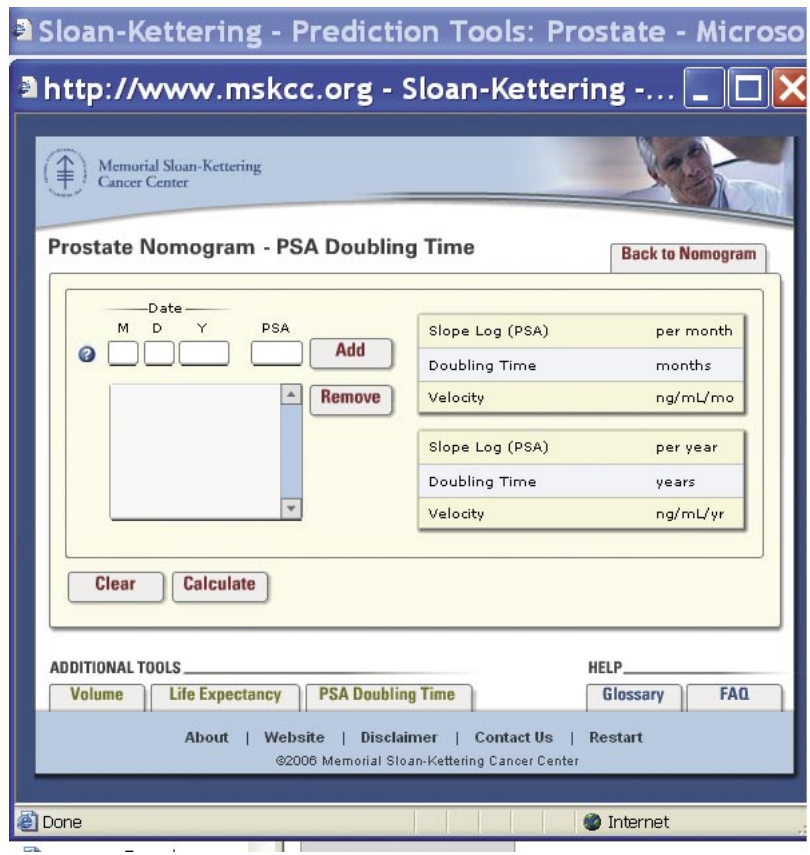

FIGURA 1. Aplicación para el cálculo de PSADT y PSAV disponible en www. mskcc.org.
La medición del PSADT está sujeta a una cierta variabilidad al no haberse establecido un único modelo matemático para obtener dicho valor. Existen diferentes maneras de calcular el PSADT. Una de ellos utiliza la pendiente de la línea que mejor se ajusta a la totalidad de los valores disponibles y otra traza esta línea entre el primer y el ultimo valor disponible de PSA. Svatek (1) compara estos dos métodos con un tercero basado la incorporación de coeficientes al azar en una muestra de 122 pacientes andrógenoindependientes, concluyendo que éste último método es más exacto. A efectos prácticos, y para su uso clínico, existe una herramienta informática descargable desde internet de manera gratuita a tráves de la página web del Memorial Sloan-Kettering Cancer Center (www.mskcc.org) que, además de incorporar diferentes nomogramas predictores, ofrece el cálculo automático del PSADT y PSAV (Figura 1).

\section{PSADT en el diagnóstico de cáncer de próstata}

La aparición del PSA en la década de los ochenta revolucionó el diagnóstico de esta neoplasia dando lugar a un explosión en su incidencia.

Ya en el comienzo de la década de los 90 se reconoció la importancia no sólo del valor absoluto del PSA sino de sus cambios relativos en el tiempo. De hecho, Ballentine Carter (2) en 1992 estudió la importancia de la velocidad del PSA en el diagnóstico de cáncer, estableciéndose el punto de corte de $0,75 \mathrm{ng} / \mathrm{ml} / \mathrm{año}$ como valor por encima del cual la probabilidad de la existencia de esta neoplasia era mayor.

\section{PSADT preoperatorio como indicador del resultado de la prostatectomía radical}

En 1997 se comenzó a evaluar el comportamiento de la variación longitudinal del PSA expresada como PSADT antes de la prostatectomía radical (3). En este estudio pionero, los autores estudian 150 pacientes que tenían, al menos, tres valores de PSA antes de ser operados encontrando una fuerte correlación entre el PSADT preoperatorio y la existencia de márgenes positivos y la afectación de vesículas seminales. Sin embargo, no logran encontrar relación entre la cinética del PSA y la existencia de recidiva bioquímica.

En un reciente estudio y utilizando en este caso la PSAV como expresión de la cinética de PSA, D'Amico (4) estudia la cinética del PSA preoperatorio como discriminador del riesgo de muerte, mediante la medida de la velocidad del PSA (PSAV) antes de la operación. Los autores han evaluado esta cuestión sobre una muestra de 1.804 pacientes 
procedentes de un programa de cribado y sometidos a prostatectomía radical. Las características singulares de esta serie en cuanto a su procedencia de un programa de screening, circunstancia a tener en cuenta en la interpretación de los resultados, se pone de manifiesto en una mediana de PSA de la muestra de $4,3 \mathrm{ng} / \mathrm{ml}$, cifra sensiblemente baja en nuestro medio clínico.

En la Tabla I puede verse que, entre las diferentes variables preoperatorias estudiadas, PSAV, Gleason de la biopsia y estadio clínico, la velocidad del PSA antes de la prostatectomía es la que tiene una vinculación más estrecha con la mortalidad por cáncer de próstata tras tratamiento quirúrgico. Esta relación viene expresada en un riesgo relativo de 9,8 para la PSAV mayor de $2 \mathrm{mg} / \mathrm{ml} /$ año frente a 3,4 y 7,4 de las otras dos variables, respectivamente.

Este papel de la PSAV preoperatorio como marcador de riesgo de mortalidad o de recidiva bioquímica ha sido confirmada por otros autores. En una reciente serie sobre 202 pacientes (5) los autores encuentran en el análisis multivariante que, tanto el Gleason como la PSAV, son factores independientes asociados con la probabilidad de supervivencia libre de recidiva bioquímica. Esta vinculación existe tanto para una PSAV de $1 \mathrm{ng} / \mathrm{ml} /$ año como para un valor de $2 \mathrm{ng} / \mathrm{ml} /$ año, aconsejando, no obstante, la utilización del valor superior ya que la asociación es mayor y un punto de corte más bajo estaría sujeto a interferencias por las fluctuaciones del PSA.

\section{PSADT como criterio de selección de los pacientes para tratamiento curativo}

Actualmente existe un importante debate en relación al sobretratamiento del cáncer de próstata localizado, lo cual ha motivado la puesta en marcha

\section{TABLA I. ANÁLISIS MULTIVARIANTE MEDIDANTE REGRESIÓN DE COX DE LOS FACTORES DE ASOCIACIÓN PREOPERATORIOS CON LA MUERTE POR CÁNCER DE PRÓSTATA TRAS PROSTATECTOMIIA RADICAL.}

\begin{tabular}{|l|c|c|}
\hline Variable & RR (IC 95\%) & P \\
\hline PSAV>2ng/ml/año & $9,8(2,8-34,3)$ & $<0,001$ \\
\hline Gleason 8-10 & $3,4(1,2-9,8)$ & 0,02 \\
\hline T2 & $7,4(2,4-22,4)$ & $<0,001$ \\
\hline
\end{tabular}

de estudios sobre la utilización de tratamiento curativo diferido en cáncer de próstata de bajo riesgo, sólo cuando se detecta progresión de la enfermedad. Además de criterios de progresión histológica tras una segunda biopsia, la cinética del PSA se ha convertido, tras lo expresado en el apartado anterior, en un criterio esencial para distinguir aquellos pacientes que pueden seguir en observación de los que deben ser aconsejados la realización de tratamiento de intención curativo. En estos, protocolos, aún experimentales, los autores con más experiencia $(6,7)$ utilizan un PSADT menor de 3 años como criterio, entre otros, de indicación de tratamiento activo.

\section{PSADT como predictor de mortalidad tras PR}

La recidiva $B Q$ tras tratamiento quirúrgico del cáncer de próstata mediante prostatectomía radical se produce en aproximadamente el $35 \%$ de los pacientes al cabo de 10 años (8). La mediana de tiempo desde que se produce la elevación de PSA hasta el desarrollo de metástasis es de 8 años y desde la aparición de metástasis hasta el fallecimiento de 5 años (9). Esta larga evolución, junto con la ausencia de estudios complementarios que puedan discernir sobre el origen del PSA cuando se produce la recidiva bioquímica por un lado, y, por otro, la ausencia de datos sobre la eficacia del tratamiento adyuvante para retrasar la muerte por cáncer, hace que el clínico tenga un difícil problema en el diagnóstico etiológico y la elección del tratamiento. Desde hace tiempo, sabemos que los pacientes más proclives a desarrollar una recidiva local son aquellos con una elevación ligera y tardía (más de 2 años) de PSA, existiendo, además factores patológicos que hacen más probable esta circunstancia como son los márgenes quirúrgicos positivos, un índice de Gleason de la pieza de prostatectomía entre 8-10. La cinética del PSA se ha utilizado en los últimos años como elemento discriminatorio de las causas de la elevación de este marcador. En un estudio sobre 379 pacientes con recidiva $B Q$ tras cirugía seguidos durante un tiempo medio de 10 años, Freedland (10) encuentra que sólo el Gleason (8-10), el tiempo transcurrido desde la cirugía (3 años) y el PSADT son variables independientes que actúan como factores de riesgo de la mortalidad específica por cáncer. Como puede verse en la Tabla II un PSADT $<3$ meses tiene un HR de 27,48 frente a 3,53 para el tiempo transcurrido y 2,26 para el Gleason, lo que convierte a esta variable como la más potente en cuanto a capacidad de predicción de la muerte por cáncer de próstata. (Tabla II).

Esta asociación entre el PSADT y la mortalidad tras prostatectomía radical ya fue observada a principios de los noventa por D'Amico (11). 


\section{PSAV como predictor de mortalidad tras radioterapia externa}

El comportamiento del PSA antes del tratamiento con radioterapia ha sido también estudiado en cuanto a su capacida de prever los resultados del tratamiento. En una serie de 358 pacientes (12) sin tratamiento hormonal neo ni adyuvante $y$ tratados con una dosis mínima de 70,35 Gy y, tras un seguimiento mediano de 4 años, una PSAV mayor de $2 \mathrm{ng} / \mathrm{ml} /$ año se asociaba con el tiempo a recidiva bioquímica y la mortalidad por cáncer de próstata con un factor de asociación (HR) de 12,0 (IC 95\%: $3,0-54,0)$ para esta variable.

Cuando se estratifican los pacientes por grupos de riesgo, esta capacidad predictora de la PSAV preoperatorio ofrece un HR de 2,4 (IC 95\%: 1,63,5 ) y de 1,08 (IC 95\%: 1,05-1,10) para la mortalidad por cáncer de próstata en pacientes de bajo y alto riesgo, respectivamente.

\section{$P S A D T$ en la selección de pacientes con recidiva $B Q$ tras PR candidatos a radioterapia externa}

Los pacientes sometidos a prostatectomía radical deben tener, idealmente, un PSA indetectable prácticamente 1 mes después de la intervención y mantenerse así a lo largo de su evolución. Desgraciadamente, esto no es siempre así y un porcentaje variable de pacientes experimentan lo que se ha venido en llamar recidiva bioquímica. No hay acuerdo en la definición de este concepto por lo que algunos autores establecen la cifra de $0,2 \mathrm{ng} / \mathrm{ml}$ como valor para determinar la presencia de recidiva y otros lo hacen en 0,4.

Una vez que se detecta la elevación del PSA tras la cirugía, el urólogo debe valorar la necesidad de establecer un tratamiento de rescate o de deprivación androgénica si se estima que la causa de la recidiva bioquímica es la presencia de metástasis. En

\section{TABLA II. FACTOR DE ASOCIACIÓN DE DIFERENTES VARIABLES CON EL RIESGO DE MUERTE POR CÁNCER TRAS PROSTATECTOMIIA RADICAL.}

\begin{tabular}{|l|c|}
\hline Variable & HR (IC 95\%) \\
\hline PSADT $<3$ meses & $27,48(10,66-70,85)$ \\
\hline Tiempo desde la cirugía <3 años & $3,53(1,59-7,84)$ \\
\hline Gleason $>8$ & $2,26(1,35-3,77)$ \\
\hline
\end{tabular}

esta disyuntiva se puede utilizar la cinética del PSA para discriminar aquellos pacientes con riesgo de progresión clínica y que serían por lo tanto candidatos a ser tratados tras la cirugía (13).

Los pacientes candidatos a ser tratados con radioterapia externa tras la cirugía son aquellos en los que se presume que el origen de la elevación del PSA es debida a una recidiva local. Stephenson (14) sobre una serie retrospectiva de 501 pacientes sometida a RXT externa tras cirugía y fallo bioquímico. El análisis multivariante reveló que el Gleason de la pieza entre 8-10, una cifra de PSA antes de la RXT $>2 \mathrm{ng} / \mathrm{ml}$, la presencia de margenes negativos, invasión de vesículas seminales y una PSADT $\leq 10$ meses eran factores predoctores independientes del tiempo a progresión bioquímica tras radioterapia de rescate.

Este mismo punto de corte de PSADT de 10 meses, fue encontrado en una importante serie de 1997 pacientes operados en Johns Hopkins sin tratamiento adyuvante de ningún tipo y con un seguimiento medio de 5 años publicado por Pound (9) encontrando que los pacientes con menor probabilidad de desarrollar metástasis son aquellos con Gleason 5-7, elevación de PSA tras más de 2 años y un PSADT> 10 meses.

\section{CONCLUSIONES}

Las variaciones del PSA en el tiempo englobadas bajo el concepto de cinética de PSA y expresadas como PSAV y PSADT se han convertido en un importante indicador del comportamiento del cáncer de próstata. Su medición, sujeta a variaciones matemáticas está aún abierta a una homogeneización y su interpretación abierta a la variaciones de las características de los pacientes estudiados.

La existencia de una PSAV pretratamiento mayor de 2,0 ng/ml/año se asocia con una mayor mortalidad por cáncer de próstata tanto tras prostatectomía radical como tras radioterapia.

Tras el tratamiento, un PSADT $<3$ meses situa a los pacientes en un mayor riego de mortalidad por cáncer de próstata lo que los hace potencial candidatos para evaluar esquemas alternativos de tratamiento adyuvante.

Un PSADT>10 meses en el contexto de un paciente con recidiva bioquímica tras prostatectomía radical sitúa la recidiva local como causa más probable de la elevación del PSA permitiendo al clínico la adopción de medidas terapéuticas más adecuadas. 


\section{BIBLIOGRAFÍA Y LECTURAS RECOMENDADAS ( ${ }^{*}$ lectura de interés $y^{* *}$ lectura fundamental)}

1. SVATEK, R.S.; SHULMAN, M.; CHOUDHARY, P.K. y cols.: "Critical anlysis of prostate-specific antigen doubling time calculation”. Cancer, 106: 1047, 2006.

2. CARTER, H.B.; PEARSON, J.D.; METTER, J.E. y cols.: "Longitudinal evaluation of prostate specific antigens levels in men with and without prostate disease". JAMA 267: 2215, 1992.

3. GOLUBOFF, E.T.; HEITJAN, D.F.; DE VRIES, G.M. y cols.: "Pretreatment prostate specific doubling times: use in patients before radical prostatectomy". J. Urol., 158: 1876, 1997.

4. D'AMICO, A.V.; CHEN, M.; ROEHL, K.A. y cols.: "Preoperative PSA velocity and the risk of death from prostate cancer after radical prostatectomy". N. Engl. J. Med., 351: 125, 2004.

5. PATEL, D.A.; PRESTI, J.C.; McNEAL, J.E. y cols.: "Preoperative PSA velocity is an independent prognostic factor for relapse after radical prostatectomy”. J. Clin. Oncol., 23: 6157, 2005.

6. KLOTZ, L.: "Active surveillance for prostate cancer: for whom?”. J. Clin. Oncol., 23: 8165, 2005.

7. HARDIE, C.; PARKER, C.; NORMAN, A. y cols.: "Early outcomes of active surveillance for localized prostate cancer”. BJU Int., 95: 956, 2005.

8. HAN, M.; PARTIN, A.W.; POUND, C.R. y cols.: "Long-term biochemical disease-free and cancerspecific survival following anatomic radical retropubic prostatectomy: 15-year John Hopkins experience". Urol. Clin, North Am., 28: 555, 2001.

9. POUND, C.R.; PARTIN, A.W.; EISENBERGER, M.A. y cols.: "Natural history of progression after PSA elevation following radical prostatectomy". JAMA, 281: 1591, 1999.

10. FREEDLAND, S.J.; HUMPHREYS, E.B.; MANGOLD, L.A. y cols.: "Risk of prostate cancer-specific mortality following biochemical recurrence after radical prostatectomy". JAMA, 294: 433, 2005.

11. D’AMICO, A.V.; HANKS, G.E.: "Linear regressive analysis using prostate-specific antigen doubling time for predicting tumor biology and clinical outcome in prostate cancer". Cancer, 72: 2638, 1993.

12. D'AMICO, A.; RENSHAW, A.A.; SUSSMAN, B. y cols.: "Pretreatment PSA velocity and risk of death from prostate cancer following external beam radiation therapy". JAMA, 294: 440, 2005.

13. PATEL, A.; DOREY, F.; FRANKLIN, J. y cols.: "Recurrence patterns after radical retropubic prostatectomy: clinical usefulness of prostate specific antigen doubling times and log slope prostate specific antigen". J. Urol., 158: 1441, 1997.

14. STEPHENSON, A.J.; SHARIAT, S.F.; ZELEFSKY, M.J. y cols.: "Salvage radiotherapy for recurrent prostate cancer after radical prostatectomy". JAMA, 291: 1325, 2004. 